\title{
Wie wählen internistische Patienten ihr Krankenhaus? Eine Querschnittstudie
}

\section{How do internal medicine patients choose their hospital? A cross-sectional study}

(c) (ㅇ) $९$

Autoren

W. de Cruppé, M.-C. Kummer, M. Geraedts

Institut

Philipps Universität Marburg, Institut für

Versorgungsforschung und Klinische Epidemiologie

Schlüsselwörter

Krankenhauswahl, Innere Medizin, Querschnittstudie, Deutschland

Key words

hospital choice, inpatient, internal medicine, cross-sectional study, Germany

online publiziert 03.12 .2021

\section{Bibliografie}

Dtsch Med Wochenschr 2022; 147: e23-e31

DOI 10.1055/a-1653-6717

ISSN 0012-0472

(C) 2021. The Author(s).

This is an open access article published by Thieme under the terms of the Creative Commons Attribution-NonDerivative-NonCommercial License, permitting copying and reproduction so long as the original work is given appropriate credit. Contents may not be used for commecial purposes, or adapted, remixed, transformed or built upon. (https://creativecommons.org/licenses/by-nc-nd/4.0/)

Georg Thieme Verlag KG, Rüdigerstraße 14,

70469 Stuttgart, Germany

Korrespondenzadresse

Dr. Werner de Cruppé

Philipps Universität Marburg, Institut für

Versorgungsforschung und Klinische Epidemiologie,

Karl-von-Frisch-Straße 4, D-35043 Marburg

decruppe@uni-marburg.de

Zusätzliches Material finden Sie unter

https://doi.org/10.1055/a-1653-6717

\section{ZUSAMMENFASSUNG}

Einleitung Die freie Krankenhauswahl durch Patienten wird gesundheitspolitisch gefördert und soll durch die Qualitätsberichte der Krankenhäuser unterstützt werden. Doch wie entscheiden sich Patienten für ein Krankenhaus? Wie viele können selber entscheiden, wo informieren sie sich und was sind in der konkreten Situation ihre Entscheidungskriterien? Dies soll für stationäre Patienten der Inneren Medizin im Vergleich zu denen anderer Fachgebiete beantwortet werden.

Methoden Die Daten entstammen einer Beobachtungsstudie. Die nach Fachgebieten und Krankenhausversorgungsstufen geschichtete Zufallsstichprobe wurde in 46 Fachabteilungen von 17 Krankenhäusern aus 15 Städten und Gemeinden Nordrhein-Westfalens erhoben. Die gewichtete Stichprobe wertet 758 Patienten der Inneren Medizin und 1168 Patienten anderer Fachgebiete deskriptiv und inferenzstatistisch aus. Ergebnisse Internistische Patienten sind älter, häufiger Männer, ohne Migrationshintergrund und chronisch krank, zudem öfter stationär vorbehandelt. Etwa die Hälfte entscheidet selbst über das Krankenhaus, wobei die eigene Kenntnis des Krankenhauses durch einen Voraufenthalt die wichtigste Informationsquelle darstellt und wichtige Entscheidungskriterien die eigene Vorerfahrung, der Ruf des Krankenhauses und die Empfehlung der ambulanten Behandler sind. Der kleine Anteil Patienten mit mehr Zeit vor der Aufnahme wählt das Krankenhaus aktiver.

Diskussion In der Inneren Medizin können weniger Patienten selbst über das Krankenhaus bestimmen. Diese entscheiden dann überwiegend aufgrund ihrer Vorerfahrung mit dem Krankenhaus und setzen die erneute Behandlung im ihnen bekannten Krankenhaus, in dem auch sie bekannt sind, fort. Ein kleiner Anteil jüngerer, gebildeterer und weniger krankenhauserfahrener Patienten informiert sich aktiver vor elektiven Eingriffen. Die Behandlungserfahrungen der Patienten sind zentral bei der eigenen Krankenhauswahl und über den sozialen Austausch auch bei der ihrer Angehörigen.

\section{ABSTRACT}

Background The free choice of hospital by patients is promoted by health policy and is to be supported by the quality reports of hospitals. But how do patients choose a hospital? How many can decide for themselves, where do they inform themselves and what are their decision criteria in the specific situation? This will be answered for inpatients of internal medicine in comparison to those of other specialties.

Methods The data originate from an observational study. The random sample, stratified by specialties and hospital care levels, was collected in 46 departments of 17 hospitals 
from 15 cities and municipalities in North Rhine-Westphalia. The weighted sample evaluates 758 patients of internal medicine and 1168 patients of other specialties descriptively and inferentially.

Results Internal medicine patients are older, more often men, without a migration background and chronically ill, and also more often pretreated as inpatients. About half decide on the hospital themselves, with their own knowledge of the hospital through a previous stay being the most important source of information and the important decision criteria being their own previous experience, the hospital call and the recommendation of outpatient treatment providers. The small propor- tion of patients with more time before admission choose more actively.

Conclusions In internal medicine, fewer patients can decide on the hospital themselves. These then decide quite predominantly on the basis of their previous experience with the hospital and continue the renewed treatment in the hospital with which they are also familiar. A small proportion of younger, more educated and less hospital-experienced patients inform themselves more actively before elective procedures. Patients' treatment experiences are central to their own and their relatives' choice of hospital via social exchange.

\section{Einleitung}

In der Inneren Medizin werden jährlich über 7 Millionen Patienten auf allen Krankenhaus-Versorgungsstufen behandelt und damit täglich durchschnittlich 20000 Patienten in den 1998 Fachabteilungen aufgenommen [1]. So versorgt die Innere Medizin $37 \%$ aller vollstationären Patienten in Deutschland und ist in der Hinsicht das größte Fachgebiet und überall im Land präsent.

Eine der gesundheitspolitischen Anforderungen an die Krankenhäuser ist, mit guter Qualität um die Patienten zu werben. Patienten wiederum werden ermuntert, ihr Krankenhaus selbst auszusuchen und dabei „auch objektive Kriterien einzubeziehen“, wie sie beispielsweise die Qualitätsberichte der Krankenhäuser enthalten [2].

Studien zur Krankenhauswahl erheben das Entscheidungsverhalten teils an Patienten vor oder nach einem Krankenhausaufenthalt, teils hypothetisch an gesunden Personen wie an Krankenversicherten, Studenten oder der Allgemeinbevölkerung [3, 4]. Patienten werden überwiegend zu spezifischen, elektiven operativen Eingriffen befragt, vor allem zu Gelenkersatz, onkologischchirurgischen Operationen, herzchirurgischen Eingriffen, kardiologischen oder anderen Katheter-Interventionen [3, 4]. Hierbei geben Patienten vor allem und über die Jahre stabil an, ihre Entscheidung in Absprache mit ihren Angehörigen und ambulanten Ärzten zu treffen. Damit folgen sie nicht den Empfehlungen und Hinweisen der Gesundheitspolitik: „Denn persönliche Einschätzungen ermöglichen keine zuverlässige Aussage über die wirkliche Qualität eines Krankenhauses. Hier lohnt sich ein Blick in die Qualitätsberichte, ..." [2]. Doch dieses gesundheitspolitische Bemühen, wissenschaftlich basierte Qualitätskennziffern in der öffentlichen Qualitätsberichterstattung den Patienten zur Verfügung zu stellen, greifen Patienten selten für ihre Krankenhauswahl auf [5-8]. Marshall bilanziert, dass Patienten sich bei medizinischen Entscheidungen nicht gemäß ökonomischer Theorien zu idealen Konsumenten verhalten, sondern solche Entscheidungen ein sozialer Prozess sind. Patienten entscheiden in ihrer Krankheitssituation emotional und sozial verbunden zusammen mit den ihnen wichtigen Vertrauenspersonen [9].

Doch wie viele Patienten entscheiden selbst über das Krankenhaus und wie viel Zeit bleibt ihnen bis zu ihrer Aufnahme? Und wie gehen sie dabei vor, wo informieren sie sich und was sind in der konkreten Aufnahmesituation ihre praktisch wichtigen Entscheidungskriterien? Gerade für Patienten der Inneren Medizin ist dies nicht bekannt, und zwar eingriffsunabhängig. Diese Fragen sollen mit Daten einer Studie zur Krankenhauswahl an einer repräsentativen Stichprobe aller vollstationär aufgenommenen Patienten in der Inneren Medizin beantwortet werden.

\section{Material und Methoden}

Die vorliegende Untersuchung basiert auf einer Querschnittstudie mit multizentrisch per Fragebogen von Krankenhauspatienten erhobenen, quantitativen Primärdaten. Die Studienstichprobe ist eine nach Fachgebieten und Krankenhaus-Versorgungsstufen geschichtete Zufallsstichprobe von je durchschnittlich 50 konsekutiv vollstationär aufgenommenen Patienten aus 46 Fachabteilungen in 17 Krankenhäusern sowie aus 15 Städten und Gemeinden Nordrhein-Westfalens. Die Stichprobe schließt 11 aufnahmestarke Fachgebiete (Innere Medizin, Chirurgie, Gynäkologie, Geburtshilfe, Pädiatrie, Psychiatrie, Orthopädie, Neurologie, Urologie, HNO und Geriatrie) ein, die im Erhebungsjahr 2012 91,9\% aller Aufnahmen in Deutschland ausmachten [10]. Die Versorgungsstufe berücksichtigt 3 Ebenen, definiert nach Bettengröße-Klassen: Grundversorgung (<200 Betten), Schwerpunktversorgung (200-499 Betten) und Maximalversorgung (>499 Betten). Je Fachgebiet sind 2 Fachabteilungen unterschiedlicher Krankenhäuser der Schwerpunkt- und 2 der Maximalversorgung eingeschlossen, die HNO ist nur auf der Maximalversorgerebene berücksichtigt. Die Grundversorgerebene umfasst die Innere Medizin und Chirurgie aus je 2 Krankenhäusern. Die Innere Medizin umfasst damit 6 Abteilungen unterschiedlicher Krankenhäuser, jeweils 2 jeder Versorgungsstufe. Die Auswertung erfolgte anhand der bundesweiten Patientenverteilung nach Fachgebieten gewichtet; die Verteilung nach Versorgungsstufe entspricht annähernd der bundesweiten Verteilung.

Die statistische Auswertung gibt deskriptiv die Häufigkeit der Merkmale zur Krankenhauswahl an und vergleicht die Patienten der Inneren Medizin mit den anderen 10 Fachgebieten bei kategorialen Merkmalen mittels Chi-Quadrat-Tests nach Pearson und t-Test für die in Minuten erfragte Wohnortnähe und das Alter in Jahren. Die berichteten p-Werte der statistischen Tests sind 2-seitige $\mathrm{p}$-Werte. Die statistischen Tests dienen einem explorativen 
Vergleich der beiden Gruppen. Signifikante Unterschiede bei einem Signifikanzniveau von 0,05 sind daher nur hypothesengenerierend und müssen zur Bestätigung an anderen Stichproben überprüft werden. Da insgesamt 35 statistische Tests an der Stichprobe durchgeführt wurden, liegt multiples Testen vor und damit die Möglichkeit einer Kumulierung des Alphafehlers. Eine Adjustierung des Signifikanzniveaus von 0,05 mit der konservativen Bonferroni-Korrektur ergibt ein adjustiertes Signifikanzniveau von 0,0014 , berechnet aus 0,05/35 statistische Tests. Die in den Tabellen berichteten p-Werte sind nicht auf das multiple Testen adjustiert. Darauf haben wir wegen des rein explorativen Charakters der Gruppenvergleiche verzichtet. Bei kleinen Fallzahlen erfolgte kein statistischer Test. In den Tabellen sind für jede Variable die Gesamtzahl auswertbarer Teilnehmerantworten als Absolutwert $(n=)$ angegeben, die Variablenausprägungen dann als prozentuale Verteilung.

Der eingesetzte Fragebogen ist die Weiterentwicklung eines Erhebungsbogens aus einer vorhergehenden Studie [4] und erhebt soziodemografische Angaben, Merkmale zur Aufnahmesituation und Krankenhauserfahrung ( $\vee$ Tab. 1), Informationsquellen ( $\triangleright$ Tab. 2) und Entscheidungskriterien ( $>$ Tab.3) der Krankenhauswahl aus Patientensicht [10]. Patienten können den Bogen selbst oder zusammen mit einem Interviewer ausfüllen. Sie sollen die für sie zutreffenden Items in den beiden Fragen zu Informationsquellen und den Entscheidungskriterien ankreuzen, müssen aber nicht jedes nichtzutreffende Item als „nichtzutreffend“ markieren. Der Sozialstatus berechnet sich aus Berufsabschluss, Berufsposition, Haushaltsgröße und -einkommen [11]; der Migrationshintergrund entspricht der Definition des Statistischen Bundesamtes beim Zensus 2011. Chronische Erkrankung ist definiert als eine mehr als 6 Monate behandlungsbedürftige Erkrankung, erhoben aus Patienten- und Stationsarztsicht. Die Erhebung führten 2 geschulte Interviewer auf den Stationen durch.

\section{Ergebnisse}

\section{Stichprobe}

Von den 2368 im Erhebungszeitraum aufgenommenen Patienten sind 1925 Patienten Teilnehmer der Studienstichprobe (GesamtTeilnahmerate 81,3\%). In der Inneren Medizin beträgt die Teilnahmerate $75,0 \%$, in den anderen Fachgebieten zusammen 83,8\%. Die häufigsten Gründe für eine Nichtteilnahme in der Inneren Medizin sind eine dauerhafte $(44,6 \%)$ oder momentane $(18,0 \%)$ Beeinträchtigung der Patienten; in den anderen Fächern umfassen diese beiden Gründe 48,9\%. Weitere Gründe sind in der Inneren Medizin (andere Fachgebiete): 19,3\% (21,5\%) Entlassung bevor kontaktierbar, 6,9\% (9,4\%) Patienten wegen Erhebungsende nicht kontaktiert, 5,6\% (6,0\%) Ablehnung der Teilnahme, 3,4\% $(9,0 \%)$ Fragebogen nicht abgegeben und 2,1\% (5,2\%) zu geringe Deutschkenntnisse. Die gewichtete Stichprobe der 1925 Teilnehmer umfasst 758 Patienten der Inneren Medizin bzw. rundungsbedingt aufgrund der Gewichtung teilweise 757 Patienten, deren Ergebnisse nachfolgend berichtet werden.

\section{Soziodemografie, Aufnahmekontext, Krankenhauserfahrung}

Patienten der Inneren Medizin sind im Vergleich zu denen anderer Fachgebiete älter, häufiger Männer, ohne Migrationshintergrund und chronisch krank ( $\triangleright$ Tab. 1). Die Verteilung nach Versorgungsstufe unterscheidet sich erwartungsgemäß, da nur noch die Chirurgie zur Regelversorgung zählt. 57,8\% der internistischen Patienten entscheiden selbst über das Krankenhaus, signifikant weniger als die 66,3\% der anderen Fächer. Berücksichtigt man auch die Nichtteilnehmer und geht realistisch davon aus, dass bei dauerhaft Beeinträchtigten andere entscheiden, und unterstellt bei allen anderen Nichtteilnehmern die gleiche Verteilung wie bei den Teilnehmern, dann sinkt der Anteil der Selbst-Entscheider auf 51,3\%. Patienten der Inneren Medizin werden mit 63,1 \% häufiger als andere Patienten am selben Tag aufgenommen. Sie sind auch krankenhauserfahrener, $65 \%$ geben mehr als 5 frühere Krankenhausaufenthalte an und 70,7\% kennen das Krankenhaus durch einen Voraufenthalt, 69,6\% davon sogar die Fachabteilung.

\section{Informationsquellen und Entscheidungskriterien}

29,3\% der Patienten informieren sich nirgendwo und 48,7\% beziehen sich nur auf ihre eigene Vorerfahrung mit dem Krankenhaus, lediglich 22,0\% informieren sich irgendwo anders ( $\triangleright$ Tab. 2). Hausärzte (10,7\%) und Angehörige (10,3\%) sind die wichtigsten externen Informationsquellen, gefolgt vom ambulanten Facharzt (7,3\%) und dem Internet (6,1\%). Von den Patienten, die selbst das Krankenhaus wählen, entscheiden 37,4\% nur nach der eigenen Vorerfahrung mit dem Krankenhaus bzw. der Fachabteilung ( $\triangleright$ Tab. 3). 61,9\% geben auch andere Kriterien an, die für sie bei der Entscheidung wichtig sind. Der gute Ruf des Krankenhauses (30,9\%), die Empfehlung ihrer ambulanten Behandler (25,6\%) und die Wohnortnähe (23,7\%) sind die 3 wichtigsten Entscheidungskriterien, gefolgt von anderen individuellen, nicht näher angegebenen Gründen (17,1\%), den Angehörigenempfehlungen $(16,4 \%)$ und der Frage, ob sich die Krankenhausärzte genug Zeit für Patienten nehmen (8,5\%). Einzelne operationalisierte Qualitätskennziffern nennen nur je $1 \%$ bis $2 \%$.

\section{Zeit vor Aufnahme}

Die Differenzierung nach der verfügbaren Zeit vor Aufnahme (Online-Supplement Tab.S 1, Tab.S 2, Tab.S 3) zeigt folgende Ergebnisse für die Patienten der Inneren Medizin: Die 8,1 \% Patienten $(n=61)$ mit mehr als einer Woche Zeit vor Aufnahme sind jünger, vor allem zwischen 40 und 69 Jahren, mit 61,7\% öfter männlich, sie gehören zu 38,3\% der Oberschicht an und 90,2\% entscheiden selbst über das Krankenhaus. 71,4\% werden von Schwerpunkt- und 28,6\% von Maximalversorgern elektiv behandelt. Die durchschnittliche Entfernung vom Zuhause ist mit 32 Fahrminuten deutlich weiter. Für 10,0\% ist es der erste Krankenhausaufenthalt, nur 42,6\% waren zuvor bereits im selben Krankenhaus und nur 30,4\% in der Fachabteilung. Von diesen aber gibt keiner eine unzufriedene Bewertung des Voraufenthaltes an. Diese Patienten informieren sich vor allem beim ambulanten Facharzt (47,5\%) und Hausarzt (23,3\%), aber auch zu 28,3\% im Internet und zu 19,7\% bei Angehörigen. Die ihnen wichtigen 
- Tab. 1 Soziodemografie, Aufnahmesituation und Krankenhaus-Vorerfahrung im Vergleich zwischen Innerer Medizin und den anderen 10 Fachgebieten.

\section{Gesamtteilnehmer $\mathbf{n}=$}

soziodemografische Angaben

Alter in Jahren $\mathrm{n}=$

Mittelwert (Jahre)

Standardabweichung

Aufteilung nach Jahreskategorien

- 0-24

- 25-29

- 30-39

- 40-49

- 50-59

- 60-69

. 70-79

- 80-89

- 90 oder älter

Geschlecht $n=$

männlich

weiblich

Migrationsstatus $\mathrm{n}=$

ohne Migrationshintergrund

mit Migrationshintergrund

sozioökonomischer Status $n=$

Oberschicht

Mittelschicht

Unterschicht

Krankenversicherung $n=$

gesetzlich versichert

gesetzlich und privat zusatzversichert

privat vollversichert

Beihilfe

Aufnahmesituation

\section{Krankenhausversorgungsstufe $n=$}

Grundversorger (<200 Betten)

Schwerpunktversorger (200-499 Betten)

Maximalversorger (>499 Betten)

Entscheider über das Krankenhaus $n=$

Patient

andere Person

davon:

- Notfallrettung

- Hausarzt

- Facharzt
Innere Medizin

758

10 andere Fachgebiete

p-Wert*

1168

\begin{tabular}{l|l}
\hline 58 & 1167 \\
\hline
\end{tabular}

67,7

52,5

23,5

15,6

$0,8 \%$

$1,2 \%$

$4,9 \%$

$6,1 \%$

$15,2 \%$

$16,8 \%$

$31,1 \%$

$19,8 \%$

$4,2 \%$

758

$52,1 \%$

$47,9 \%$

758

$86,7 \%$

$13,3 \%$

743

$9,3 \%$

$41,0 \%$

$49,7 \%$

758

$92,7 \%$

$3,4 \%$

$3,4 \%$

$0,4 \%$

$14,2 \%$

$5,2 \%$

$9,9 \%$

$11,7 \%$

$14,7 \%$

$13,5 \%$

$19,8 \%$

$9,3 \%$

$1,8 \%$

1167

$40,4 \%$

$59,6 \%$

1147

$78,4 \%$

$21,6 \%$

1101

$11,3 \%$

$40,5 \%$

$47,2 \%$

1162

$87,7 \%$

0,004

$5,9 \%$

$5,3 \%$

$1,0 \%$

$<0,001$

$<0,001$

$<0,001$

0,127

\begin{tabular}{|l|l|}
\hline 758 & 1168 \\
\hline
\end{tabular}

$31,6 \%$

$11,0 \%$

$<0,001$

$37,6 \%$

$43,0 \%$

$46,0 \%$

1161

$66,3 \%$

$33,7 \%$

$42,2 \%$

$16,0 \%$

$10,0 \%$

$12,2 \%$

$6,2 \%$

$5,7 \%$

$8,7 \%$ 
- Tab. 1 (Fortsetzung)

\begin{tabular}{|c|c|c|c|}
\hline & Innere Medizin & 10 andere Fachgebiete & p-Wert* \\
\hline Gesamtteilnehmer $\mathbf{n}=$ & 758 & 1168 & \\
\hline - Krankenhausambulanz & 1,9\% & $4,1 \%$ & \\
\hline - Angehörige & $3,4 \%$ & $2,1 \%$ & \\
\hline - Sonstige & $3,0 \%$ & $2,6 \%$ & \\
\hline Zeit vor Aufnahme $n=$ & 757 & 1163 & \\
\hline Aufnahme am gleichen Tag & $63,1 \%$ & $43,2 \%$ & \multirow[t]{6}{*}{$<0,001$} \\
\hline Aufnahme am nächsten Tag & $4,2 \%$ & $5,1 \%$ & \\
\hline 2-7 Tage & $24,3 \%$ & $21,6 \%$ & \\
\hline 7-28 Tage & $7,9 \%$ & $20,4 \%$ & \\
\hline mehr als 4 Wochen & $0,0 \%$ & $9,3 \%$ & \\
\hline ich weiß nicht & $0,4 \%$ & $0,5 \%$ & \\
\hline Wochentag der Aufnahme $n=$ & 758 & 1161 & \\
\hline Montag & $22,4 \%$ & $21,3 \%$ & \multirow[t]{7}{*}{0,581} \\
\hline Dienstag & $17,4 \%$ & $18,6 \%$ & \\
\hline Mittwoch & $17,2 \%$ & $17,7 \%$ & \\
\hline Donnerstag & $16,4 \%$ & $15,3 \%$ & \\
\hline Freitag & $11,7 \%$ & $13,0 \%$ & \\
\hline Samstag & $7,7 \%$ & $5,8 \%$ & \\
\hline Sonntag & $7,3 \%$ & $8,3 \%$ & \\
\hline Entfernung vom Zuhause bis zum Krankenhaus in Fahrminuten $\mathrm{n}=$ & 754 & 1145 & \\
\hline Mittelwert (Minuten) & 19,9 & 22,3 & \multirow[t]{2}{*}{0,002} \\
\hline Standardabweichung & 15,4 & 17,4 & \\
\hline Aufnahmesituation $n=$ & 757 & 1168 & \\
\hline akut & $65,4 \%$ & $50,4 \%$ & \multirow[t]{2}{*}{$<0,001$} \\
\hline elektiv & $34,6 \%$ & $49,6 \%$ & \\
\hline chronische Erkrankung $n=$ & 758 & 1168 & \\
\hline ja & $92,3 \%$ & $67,2 \%$ & \multirow[t]{2}{*}{$<0,001$} \\
\hline nein & $7,7 \%$ & $32,8 \%$ & \\
\hline \multicolumn{4}{|l|}{ Krankenhausvorerfahrung } \\
\hline Anzahl vorheriger $\mathrm{KH}$-Aufenthalte $\mathrm{n}=$ & 758 & 1167 & \\
\hline noch nie & $1,8 \%$ & $9,3 \%$ & \multirow[t]{3}{*}{$<0,001$} \\
\hline 1 - bis 5 -mal & $33,2 \%$ & $47,5 \%$ & \\
\hline öfter als 5-mal & $65,0 \%$ & $43,2 \%$ & \\
\hline Zeitpunkt des letzten KH-Aufenthalts $n=$ & 754 & 1156 & \\
\hline in den letzten 12 Monaten & $50,8 \%$ & $45,3 \%$ & \multirow[t]{2}{*}{0,019} \\
\hline nie oder vor mehr als 12 Monaten & $49,2 \%$ & $54,7 \%$ & \\
\hline Voraufenthalt in diesem Krankenhaus $n=$ & 758 & 1167 & \\
\hline ja & $70,7 \%$ & $60,5 \%$ & \multirow[t]{2}{*}{$<0,001$} \\
\hline nein & $29,3 \%$ & $39,5 \%$ & \\
\hline $\begin{array}{l}\text { Voraufenthalt in dieser Fachabteilung } \\
\text { (bei Voraufenthalt in diesem Krankenhaus) } n=\end{array}$ & 530 & 684 & \\
\hline ja & $69,6 \%$ & $66,2 \%$ & \multirow[t]{2}{*}{0,210} \\
\hline nein & $30,4 \%$ & $33,8 \%$ & \\
\hline
\end{tabular}


- Tab. 1 (Fortsetzung)

\section{Gesamtteilnehmer $\mathbf{n}=$}

Zufriedenheit mit diesem Krankenhaus beim letzten Aufenthalt

$\mathrm{n}=$

sehr zufrieden

zufrieden

weniger zufrieden

nicht zufrieden

\section{Innere Medizin}

758

527

$44,8 \%$

$50,3 \%$

$4,4 \%$

$0,6 \%$
10 andere Fachgebiete

1168

692

$37,1 \%$

$56,8 \%$

$5,3 \%$

$0,7 \%$
p-Wert ${ }^{*}$

0,061

2-seitig, t-Test bei ,Alter in Jahren` und ,Entfernung vom Zuhause zum Krankenhaus`, übrige Variablen mit Chi-Quadrat-Test.

- Tab. 2 Vergleich der von Patienten über Krankenhäuser genutzten Informationsquellen zwischen Innerer Medizin und den anderen 10 Fachgebieten.

\begin{tabular}{|c|c|c|c|}
\hline & Innere Medizin & 10 andere Fachgebiete & p-Wert* \\
\hline & $n=757$ & $n=1168$ & \\
\hline genutzte Informationsquelle & $\%$ & $\%$ & \\
\hline nirgendwo informiert & 29,3 & 22,9 & 0,002 \\
\hline $\begin{array}{l}\text { ausschließlich eigene Kenntnis des Krankenhauses } \\
\text { (keine Angabe externer Informationsquellen) }\end{array}$ & 48,7 & 41,1 & 0,001 \\
\hline eine oder mehrere externe Informationsquellen & 22,0 & 36,0 & $<0,001$ \\
\hline externe Informationsquellen, aufgeschlüsselt (Mehrfachangaben): & $\%$ von 757 & $\%$ von 1168 & \\
\hline Angehörige & 10,3 & 16,8 & $<0,001$ \\
\hline Facharzt & 7,3 & 14,6 & $<0,001$ \\
\hline Hausarzt & 10,7 & 10,2 & 0,731 \\
\hline Internet & 6,1 & 11,1 & $<0,001$ \\
\hline Krankenhausambulanz & 2,6 & 5,9 & 0,001 \\
\hline Krankenhausbesichtigung & 0,0 & 2,8 & \\
\hline Informationsveranstaltung des Krankenhauses & 0,4 & 2,1 & \\
\hline andere Informationsquellen & 0,8 & 1,5 & \\
\hline Informationsbroschüren & 0,4 & 1,5 & \\
\hline Tageszeitungen & 0,4 & 0,3 & \\
\hline Krankenkassen & 0,4 & 0,2 & \\
\hline Patientenverbände & 0,0 & 0,1 & \\
\hline Selbsthilfegruppen & 0,0 & 0,1 & \\
\hline Verbraucherberatungen & 0,0 & 0,0 & \\
\hline
\end{tabular}

Entscheidungskriterien sind die Empfehlung der eigenen ambulanten Behandler (63,6\%), der gute Ruf des Krankenhauses (52,7\%), und 2 Aspekte der Arzt-Patienten-Beziehung im Kran- kenhaus, nämlich, ob sich die Ärzte dort genug Zeit für Patienten nehmen und diese in Entscheidungen einbeziehen, mit jeweils $21,8 \%$. 
- Tab. 3 Vergleich der wichtigen Entscheidungskriterien der Patienten, die selbst über das Krankenhaus entscheiden, zwischen Innerer Medizin und den anderen 10 Fachgebieten.

\begin{tabular}{|c|c|c|c|}
\hline & Innere Medizin & 10 andere Fachgebiete & p-Wert ${ }^{*}$ \\
\hline & $n=438$ & $n=770$ & \\
\hline Entscheidungskriterium & $\%$ & $\%$ & \\
\hline keine Kriterien angegeben & 0,7 & 1,6 & 0,188 \\
\hline ausschließlich eigene Erfahrung ohne Angabe weiterer Kriterien & 37,4 & 31,2 & 0,027 \\
\hline eine oder mehrere Angaben zu weiteren Kriterien & 61,9 & 67,2 & 0,064 \\
\hline weitere Kriterien, aufgeschlüsselt (Mehrfachnennung): & $\%$ von 438 & $\%$ von 770 & \\
\hline guter Ruf des Krankenhauses & 30,9 & 29,7 & 0,675 \\
\hline Empfehlung der ambulant behandelnden Ärzte (Hausarzt, Facharzt) & 25,6 & 31,9 & 0,023 \\
\hline Entfernung des Krankenhauses von zu Hause & 23,7 & 25,7 & 0,447 \\
\hline Empfehlung von Angehörigen & 16,4 & 23,4 & 0,004 \\
\hline ob Ärzte sich für die Patienten genug Zeit nehmen & 10,5 & 15,3 & 0,018 \\
\hline andere Gründe & 17,1 & 9,2 & $<0,001$ \\
\hline ob Patienten bei Entscheidungen zur Behandlung mit einbezogen werden & 8,5 & 10,3 & 0,310 \\
\hline $\begin{array}{l}\text { ob die medizinisch-technische Ausstattung des Krankenhauses auf dem neuesten } \\
\text { Stand ist }\end{array}$ & 2,1 & 8,8 & \\
\hline $\begin{array}{l}\text { wie die Behandlungserfolge des Krankenhauses bei der für mich notwendigen } \\
\text { Behandlung sind }\end{array}$ & 0,7 & 5,6 & \\
\hline wie das Krankenhaus mit öffentlichen Verkehrsmitteln erreichbar ist & 1,4 & 4,8 & \\
\hline ob das Krankenhaus alle hygienischen Regeln einhält & 0,0 & 4,8 & \\
\hline wie lang die Wartezeiten bis zur Aufnahme sind & 0,7 & 4,2 & \\
\hline wie oft das Krankenhaus die für mich nötige Behandlung durchführt & 0,0 & 4,2 & \\
\hline wie andere Patienten das Krankenhaus in einer Zufriedenheitsbefragung bewerten & 1,4 & 2,3 & \\
\hline wie häufig Komplikationen nach der für mich nötigen Behandlung auftreten & 0,7 & 2,1 & \\
\hline ob das Krankenhaus nach medizinischen Leitlinien behandelt & 0,7 & 2,1 & \\
\hline wie häufig Entzündungen bei Patienten im Krankenhaus auftreten & 0,7 & 1,7 & \\
\hline wie viele Patienten bei der für mich nötigen Behandlung versterben & 0,0 & 0,9 & \\
\hline
\end{tabular}

\section{Diskussion}

In der Inneren Medizin werden nicht nur die meisten vollstationären Patienten behandelt, diese sind auch älter, öfter chronisch erkrankt und werden viel öfter akut aufgenommen. Zudem waren sie bereits häufiger in stationärer Behandlung als Patienten der anderen Fachgebiete und kennen zu 70 \% das Krankenhaus schon durch mindestens einen Voraufenthalt und von diesen kennen ebenso viele auch die internistische Abteilung, Entsprechend dem höheren Alter und der häufigeren akuten Aufnahmesituation entscheiden nur $58 \%$ selbst über das Krankenhaus und damit deutlich weniger als die zwei Drittel in den anderen Fachgebieten. Bei diesem Ergebnis sollte aber auch der in der Inneren Medizin mit 25 \% höchste Anteil an Nichtstudien-Teilnehmern mitbetrachtet werden, denn dann reduziert sich die Zahl selbst entscheidender Patienten auf knapp die Hälfte.
Bereits dieses Aufnahmeprofil der internistischen Patienten kennzeichnet ihr Vorgehen bei der Krankenhauswahl. Zentral als Informationsquelle ist die eigene Vorkenntnis über Krankenhäuser, ergänzt um ihre ambulanten Behandler, den Hausarzt und den spezialisierten Facharzt. Die wichtigsten, praktisch entscheidungsrelevanten Kriterien sind für Patienten die eigene Vorerfahrung mit dem Krankenhaus und die Empfehlungen der Angehörigen sowie ihres Haus- und spezialisierten Facharztes als Vertrauenspersonen, zudem der Ruf des Krankenhauses und die Wohnortnähe. Damit entsprechen die Ergebnisse zur Krankenhauswahl den häufig gefundenen Studienergebnissen [3] und bestärken den von Marshall in seiner Übersicht resümierten [9] sozialen Prozesscharakter, der das Entscheidungsverhalten der Patienten kennzeichnet. Gleichzeitig verdeutlichen die Ergebnisse, dass Patienten die gesundheitspolitisch ihnen anempfohlene Orientierung an „objektiven Kriterien“, wie sie die Qualitätsbericht- 
sangaben enthalten, kaum befolgen [2]. Doch ein kleinerer Anteil von $8 \%$ der Patienten sticht beim Entscheidungsverhalten hervor. Diese Patienten kommen zu elektiven Eingriffen in die internistischen Fachabteilungen der Schwerpunkt- und Maximalversorgung. Sie sind im Alter von 40 bis Ende Sechzig jünger, öfter männlich und gehören häufiger der Oberschicht an. Zudem waren sie seltener oder zuvor gar nicht in stationärer Behandlung und kennen das Krankenhaus bzw. die Fachabteilung seltener, doch wenn, dann war niemand von ihnen mit dem Voraufenthalt unzufrieden. Gemäß diesem Aufnahmeprofil informieren sich diese Patienten vor dem anstehenden Eingriff in einer eher früheren Krankheitsphase häufiger bei externen Informationsquellen, vor allem ihrem spezialisierten Facharzt, im Internet, beim Hausarzt und auch in der Krankenhausambulanz. Entscheidungsrelevant sind für sie die professionelle Empfehlung ihrer Behandler, der Ruf des Krankenhauses und 2 Kriterien der antizipierten Arzt-Patienten-Beziehung im Krankenhaus, nämlich einmal, dass sich die Ärzte dort genug Zeit für die Patienten nehmen und zum anderen, dass sie diese in die Behandlungsentscheidungen einbeziehen. Einige von ihnen geben zudem objektive Qualitätskriterien als relevant an. Somit befolgt diese Teilgruppe ein klein wenig das gesundheitspolitisch empfohlene, wissenschaftlich basierte Vorgehen, objektive Qualitätskriterien bei der Krankenhauswahl zu berücksichtigen. Doch passt dieses Vorgehen der jüngeren und gebildeteren Patienten in einer eher frühen Krankheitsphase auch zu einem - mangels eigener Vorerfahrung - aktiveren Suchverhalten in alle Informationsrichtungen, wobei beziehungsvermittelte Informationen klar entscheidend sind.

Aus Krankenhaus- und Abteilungssicht sind somit in der Inneren Medizin 3 Patientengruppen im Hinblick auf die Krankenhauswahl unterscheidbar: Knapp die Hälfte der Patienten entscheidet nicht selbst, sondern das tun die Notfallrettung oder ambulante Behandler als Einweiser. Dann folgt eine größere Gruppe von gut $40 \%$, die sich ganz überwiegend nach der eigenen Vorerfahrung mit dem Krankenhaus bzw. der Fachabteilung für die erneute Behandlung im selben Krankenhaus entscheidet und schließlich gibt es noch eine kleinere Patientengruppe von knapp $10 \%$ mit mehr Zeit vor der Aufnahme, die sich aktiver und umfänglicher entscheidet.

Insgesamt zeigen die Ergebnisse, wie stark die Patienten in der Inneren Medizin mit ihrem Behandlungsort und ihren stationären Behandlern verbunden sind. Die hohe Anzahl älterer Patienten mit chronischen Erkrankungen und zahlreichen Krankenhaus-Voraufenthalten verdeutlicht, dass Patienten im Krankheitsverlauf immer wieder stationärer Behandlung bedürfen, diese oft akut oder mit sehr kurzem Zeitvorlauf aufgenommen werden und dann in das ihnen vorbekannte Krankenhaus gehen.

Für die internistischen Abteilungen bedeutet dies: Zu einem großen Teil kommen Patienten bei einer erneut nötigen Behandlung bei eigener Entscheidung oder der ihrer Einweiser dort wieder zur Aufnahme, wo sie mit ihrem individuellen Krankheitsverlauf bekannt sind und wo sie auch die ärztlichen und pflegerischen Behandler kennen und sich somit eine vertrauensvolle Beziehung aufgebaut hat.

Der Erfahrung, die Patienten im Verlaufe ihrer Behandlung gemacht haben, kommt in mehrfacher Hinsicht eine wichtige Bedeutung zu. Patienten nehmen diese nicht nur als Kranken-
haus-Vorerfahrung für eigene zukünftige Entscheidungen mit. Sie ist auch das, was über Angehörige und ambulante Behandler im sozialen Prozess der Krankenhausentscheidung dann auch für Angehörige im Falle einer bevorstehenden Krankenhausaufnahme entscheidungswirksam wird -dies insbesondere bei denen, die jünger sind und noch in der diagnostischen Abklärungsphase oder der ersten Therapiephase ihres Krankheitsverlaufs stehen. Und letztlich formt der Austausch über die gemachten Erfahrungen dann auch den Ruf eines Krankenhauses.

In der Behandlungserfahrung verbinden sich somit sowohl die medizinische Qualität der Behandlung, die Zufriedenheit der einzelnen Patienten, als auch der soziale Austausch über das Krankenhaus und formen zusammen die Entscheidung der Patienten, sich dort in Behandlung zu begeben.

\section{Limitationen}

Die Studienstichprobe besteht aus zufallsgezogenen Patienten aus 6 internistischen Fachabteilungen, doch kann die Patientenzusammensetzung in internistischen Fachabteilungen mit eingriffsspezifischen Schwerpunkten und einem höheren Anteil elektiver Patienten andere Verteilungsmuster der Patientengruppen und damit der Krankenhauswahl bedingen. Die Studie ist eine Beobachtungsstudie und die vorgestellten Ergebnisse einschließlich der statistischen Testergebnisse sind als explorativ anzusehen, und müssen durch weitere Untersuchungen bestätigt werden.

\section{KERNAUSSAGEN}

- Patienten in der Inneren Medizin sind älter, öfter chronisch krank und kommen häufiger akut zur Aufnahme als Patienten der anderen Fachgebiete.

- Etwas mehr als die Hälfte der Patienten entscheidet selbst über das Krankenhaus, das ist damit deutlich weniger als in den anderen Fachgebieten.

- Die eigene Kenntnis des Krankenhauses ist für die Hälfte der Patienten die wichtigste Informationsquelle, für $18 \%$ auch ihre Haus- und anderen Fachärzte.

- Für die meisten Patienten, die selbst entscheiden, ist ihre eigene Krankenhaus-Vorerfahrung das wichtigste Entscheidungskriterium, gefolgt vom Ruf des Krankenhauses, den Empfehlungen der Behandler und der Wohnortnähe.

- Etwa $10 \%$ der Patienten haben mehr als eine Woche Zeit vor Aufnahme und kommen auf der Schwerpunkt- und Maximalversorgerebene zur elektiven Behandlung; sie sind jünger, häufiger männlich, gehören öfter der Oberschicht an und entscheiden nach einem aktiveren Wahlverhalten selbst über das Krankenhaus.

\section{Einhaltung ethischer Richtlinien}

Die Studie erhielt am 18.10.2011 ein positives Votum der Ethikkommission der Universität Witten/Herdecke (Nr. 95/2010). Die Studie wurde gemäß der Deklaration von Helsinki in der Revision von 2008 durchgeführt und alle beteiligten Patienten gaben nach 
Aufklärung über die Studie ihr schriftliches Einverständnis zur Teilnahme an der Fragebogenerhebung.

\section{Finanzierung der Studie}

Die Studie wurde vom Bundesministerium für Bildung und Forschung unter dem Kennzeichen 01GX1047 im Rahmen des Förderschwerpunktes „Versorgungsnahe Forschung - Patientenorientierung und chronische Krankheiten “ gefördert.

\section{Interessenkonflikt}

Die Autorinnen/Autoren geben an, dass kein Interessenkonflikt besteht.

\section{Danksagung}

Wir bedanken uns bei allen Patienten für ihre mitgeteilten Erfahrungen, den Stationsmitarbeitern sowie den Abteilungsleitern für ihre Unterstützung. Dem Bundesministerium für Bildung und Forschung danken wir für die Förderung (01GX1047) im Rahmen des Förderschwerpunktes „Versorgungsnahe Forschung - Patientenorientierung und chronische Krankheiten“.

\section{Literatur}

[1] Statistisches Bundesamt (Destatis). Fachserie 12 Reihe 6.1.1 Gesundheit, Grunddaten der Krankenhäuser 2019. Statistisches Bundesamt, Wiesbaden. 2021 https://www.statistischebibliothek.de/mir/receive/DESerie_ mods_00000124; Zugriff: 10.7.2021
[2] Bundesministerium für Gesundheit. Ratgeber Krankenhaus. Druck und Verlagshaus Zarbock, Frankfurt am Main. 2021 https://www.bundesge sundheitsministerium.de/service/publikationen/gesundheit/details. html?bmg\%5Bpubid\%5D=2958; Zugriff 10.7.2021

[3] Victoor A, Delnoij DM, Friele RD et al. Determinants of patient choice of healthcare providers: a scoping review. BMC Health Serv Res 2012; 12: 272

[4] de Cruppé W, Geraedts M. Wie wählen Patienten ein Krankenhaus für elektive operative Eingriffe? Bundesgesundheitsblatt Gesundheitsforschung Gesundheitsschutz 2011; 54: 951-957

[5] Ketelaar NA, Faber M], Flottorp S et al. Public release of performance data in changing the behaviour of healthcare consumers, professionals or organisations. Cochrane Database Syst Rev 2011; 11: 1465-1858

[6] Metcalfe $D$ et al. Impact of public release of performance data on the behaviour of healthcare consumers and providers. Cochrane Database Syst Rev 2018; 9: 1465-1858

[7] Fung CH, Lim Y-W, Mattke $S$ et al. Systematic review: the evidence that publishing patient care performance data improves quality of care. Ann Intern Med 2008; 148: 111-123

[8] Faber M, Bosch M, Wollersheim $\mathrm{H}$ et al. Public reporting in health care: how do consumers use quality-of-care information? A systematic review. Med Care 2009; 47: 1

[9] Marshall M, McLoughlin V. How do patients use information on health providers? BM] 2010; Bd. 341: c5272

[10] de Cruppé W, Geraedts M. Hospital choice in Germany from the patient's perspective: a cross-sectional study. BMC Health Services Research 2017; 17: 720

[11] Böcken J, Braun B, Repschläger U. Anhang: Messung der Sozialschichtzugehörigkeit. In: Braun B, Repschläger U, Hrsg. Gesundheitsmonitor 2012. Bürgerorientierung im Gesundheitswesen. Gütersloh: Bertelsmann Stiftung; 2013: 271-272 